\title{
Veronika Pasynkova
}

Institut de sociologie de l'Académie des Sciences de Russie (RAN)

\section{(In)dépendance et impact des nouveaux médias russes : l'exemple de la couverture des Jeux Olympiques de Sotchi de 2014}

Début 2014, les Jeux Olympiques de Sotchi dominent largement le paysage médiatique russe. Le discours médiatique élaboré autour de cet événement se présente dès lors comme un cas d'étude particulièrement intéressant pour explorer la façon dont fonctionnent les nouvelles technologies de l'information et de la communication et leur effet sur l'opinion publique russe.

Le contrôle exercé par les autorités russes sur les médias traditionnels, à savoir les chaînes de télévision et la presse écrite, a déjà fait l'objet de nombreux travaux académiques (Koltsova 2006 ; Oates 2007) : le système des médias y est souvent défini comme « néo-autoritaire », caractérisé par un contrôle étatique qui s'exerce via d'importantes barrières institutionnelles faisant obstacle à la liberté d'expression, et par l'utilisation des médias comme moyen de propagande politique (Becker 2004 ; Lee 2008).

Toutefois, les médias traditionnels ne constituent pas le seul canal de diffusion d'un discours contrôlé en Russie : ce dernier se retrouve également au sein des nouveaux

1 Cet article a été traduit et adapté du russe par Sophie Voisin, Ekaterina Gloriozova et Aude Merlin. 
médias présents sur Internet ${ }^{2}$. A partir de 2010, les autorités russes accordent en effet un intérêt croissant à la sphère du web, prenant conscience qu'il ne s'agit plus d'une source d'information marginale par rapport à la télévision. En 2014, l'adoption d'une série de lois ou d'amendements concernant en premier lieu les médias en ligne ${ }^{3}$, le départ de la rédactrice en chef du site d'information Lenta.ru ${ }^{4}$ ou du fondateur du

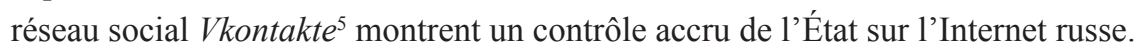

Les tentatives récentes d'instaurer un contrôle politique direct sur la sphère internet se heurtent cependant à la difficulté de maîtriser l'ensemble de la production discursive des médias en ligne. En effet, grâce à une utilisation largement répandue des blogs et réseaux sociaux, les nouveaux médias russes parviennent à transmettre une diversité de discours non officiels, assurant ainsi un pluralisme de l'information au sein du système néo-autoritaire.

Selon cette logique, en reprenant le tableau sur la régulation des médias proposé par Brett Hutchins et David Rowe pour analyser le rôle des nouveaux médias (Hutchins et Rowe 2012 : 44-45), nous représenterons les médias russes en fonction de deux variables : le caractère interactif des médias $^{6}$ (axe vertical) et le contrôle des contenus (axe horizontal) (cf. graphique 1). Les médias traditionnels se placent dans le carré 1 : non interactifs et contrôlés, tandis que les nouveaux médias, basés sur les nouvelles technologies avec des modalités souvent interactives et difficilement contrôlables (au moins au début) se placent dans le carré 4 .

2 Les médias traditionnels regroupent la presse périodique, la radio et la télévision. De leur côté, les nouveaux médias rassemblent aussi bien les moyens d'information de masse traditionnels diffusés sur Internet (publications, radio et télévision sur Internet) que les nouvelles sources d'information spécifiques à Internet : sites d'information, réseaux sociaux et blogs. Voir infra, note 4 pour les différences au sein des nouveaux médias.

3 En 2014, la Douma adopte une série de lois ou d'amendements qui multiplient les contraintes pour les journalistes : amendements à la loi contre l'extrémisme, lois contre le piratage sur Internet et contre l'emploi de l'argot dans les médias, loi protégeant les enfants ou assimilant les blogs à des médias (Daucé 2014a : 28-29).

4 Galina Timchenko a été licenciée après la publication d'un entretien avec un militant du groupe radical ukrainien Pravyj Sektor (Daucé 2014b).

5 Pavel Dourov a démissionné de ses fonctions en avril 2014 après avoir refusé de donner aux services de sécurité russes les coordonnées d'un groupe pro-Maïdan présent sur son réseau (Daucé 2014b).

6 Un média est interactif lorsque les utilisateurs peuvent intervenir, participer et donc être producteurs ou coproducteurs de l'information. Dans le cas inverse des médias non interactifs, le contenu n'est pas modifiable par les utilisateurs. 


\section{Graphique 1}

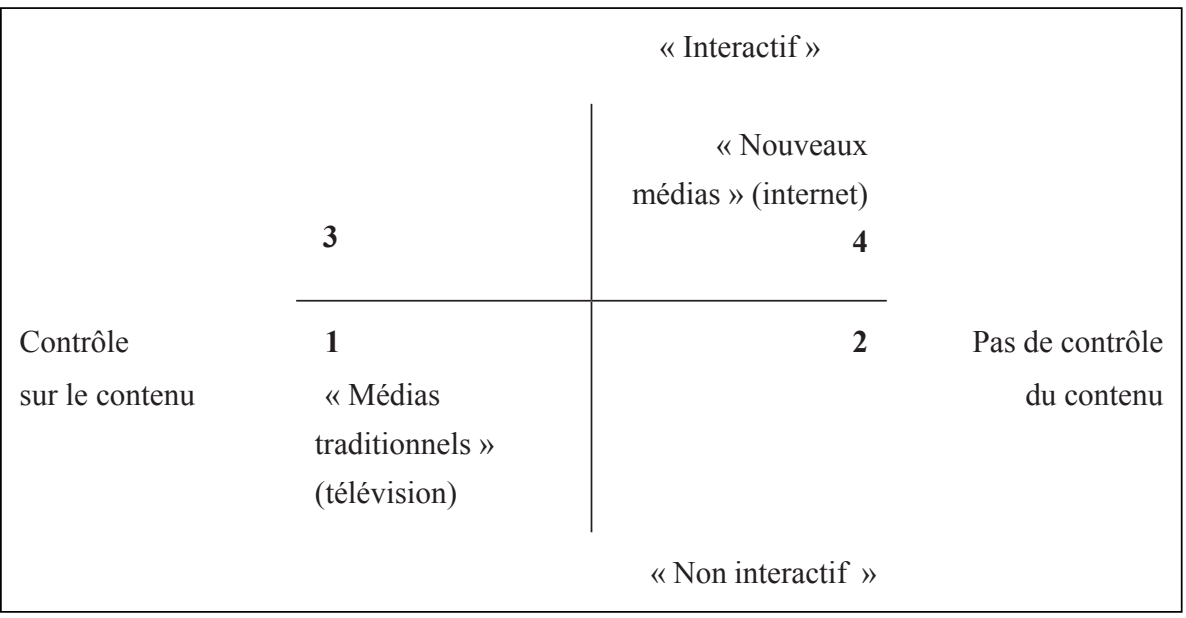

Selon les données de la Fondation pour l'opinion publique (FOM'), en juin 2014, la part d'utilisateurs actifs d'Internet s'élevait à $50 \%$ de la population adulte en Russie, ce qui représente environ 58 millions de personnes (FOM 2014). Cependant, pour les chercheurs du Centre Levada, la Russie reste un «pays de téléspectateurs » : en effet, leurs études montrent qu'environ $90 \%$ des citoyens russes s'informent via des sources télévisuelles, environ $55 \%$ n'utilisent pas de médias alternatifs et seulement $24 \%$ recourent à Internet pour s'informer (Centre Levada 2014). On peut donc constater un décalage entre d'un côté, la popularité grandissante d'Internet et, de l'autre, le faible pourcentage de consommateurs de médias sur Internet. Cette différence s'explique par la préférence des utilisateurs d'Internet pour les médias sociaux ${ }^{8}$ face aux sites d'information. En effet, d'après le Centre Levada, en 2014, plus de la moitié de la population russe utilisait activement les médias sociaux. Ainsi, pour le mois d'avril

$7 \quad<$ Fom.ru $>$

8 Les médias sociaux désignent généralement l'ensemble des sites et plateformes web médiatiques qui proposent des fonctionnalités dites "sociales » aux utilisateurs : création collaborative de contenus (wikis), échange d'information entre individus (forums, blogs ouverts aux commentaires), partage de contenus (articles, photos, vidéos, messages). Contrairement aux médias sociaux dont la fonction centrale demeure la transmission d'informations, les réseaux sociaux constituent quant à eux des plateformes dont l'objectif principal est de mettre en lien des individus. $(<\mathrm{http}: / /$ www.dictionnaireduweb.com/>). 
2014, les médias sociaux Vkontakte ${ }^{9}$, Odnoklassniki ${ }^{10}$ et Facebook ont respectivement enregistré 52,1 millions, 41 millions et 23,8 millions de visiteurs en Russie (Centre Levada 2014).

En Russie, le discours non officiel reste confiné au sein des nouveaux médias, tandis que le discours officiel ${ }^{11}$ est relayé par les médias de masse traditionnels et les réseaux sociaux s'y rattachant. En posant la question de la perméabilité entre les deux types de discours médiatiques, cet article se propose d'explorer les médias sociaux en tant que point de convergence entre les discours officiel et non officiel sur les Jeux Olympiques de Sotchi.

Ainsi, dans la première partie de l'article, nous interrogeons les Jeux Olympiques contemporains comme "événement médiatique » mondial ainsi que le rôle des nouveaux médias dans la couverture d'un tel événement. La deuxième partie est consacrée à l'analyse du discours officiel accessible sur Internet, tant sur le site officiel des Jeux qu'au sein des communautés virtuelles ${ }^{12}$ sur Vkontakte. La troisième partie aborde quant à elle le discours non officiel sur les JO, que l'on trouve sur des sites internet alternatifs, et également sur Vkontakte. Enfin, nous nous tournons vers d'autres types de données pour compléter notre analyse. Nous nous référons donc à des sondages sur les JO menés auprès de la population russe. Une analyse comparative nous permet dès lors d'appréhender les mécanismes à l'œuvre qui, d'une part, permettent aux discours officiels et non officiels d'influer sur l'opinion publique et, d'autre part, contribuent à limiter l'interactivité et à contrôler les contenus.

9 Dont le nom signifie « en contact », équivalent russe de Facebook - NdT. Le réseau social Vkontakte est la ressource internet russophone la plus visitée. Sa connection journalière atteint 64 millions d'utilisateurs, russes et internationaux (Vkontakte 2014).

10 Dont le nom signifie « Camarades de classe »- NdT.

11 Dans cet article, le discours officiel désigne le discours présent au sein des médias créés et reconnus par des instances dirigeantes (ici le Ministère des sports de la Fédération de Russie). Dans le cadre de cette recherche, il s'agit du discours présent sur le site officiel du Comité d'organisation des JO (<www.sochi2014.com $>$ ) et du groupe officiel des JO sur Vkontakte (http://vk.com/sochi2014) Voir infra sur les détails concernant les contextes de production de ce discours.

12 Les communautés virtuelles sont le plus souvent appréhendées comme un sousensemble des réseaux sociaux, reliant des individus rassemblés par un même intérêt. Pour une analyse plus approfondie de la notion, voir Proulx et Latzko-Toth 2000 et Proulx 2006. 


\section{Les JO, un événement hautement médiatique}

\section{$1.1 \mathrm{Du}$ « méga » au « média-événement »}

Les Jeux Olympiques sont un événement d'envergure mondiale, du fait de sa taille et de sa portée médiatique (Roche 2000 ; Jennings 2012), ainsi que de par ses retombées socio-économiques. Pour John Horne, les JO peuvent être définis comme un « mégaévénement » aux conséquences importantes en termes d'image et de développement économique, tant pour la ville que pour la région et le pays où ils se déroulent (Horne 2007 : 81-82). Pour Maurice Roche, c'est surtout le caractère international (qu'il s'agisse tant des participants que des spectateurs) des JO qui en fait un mégaévénement (Roche 2000 : 4). L'audience dont jouissent les JO étant tributaire d'une large couverture médiatique à l'échelle mondiale, on peut aussi définir les JO comme un «média-événement».

Sur la scène mondiale, les Jeux Olympiques sont devenus un enjeu médiatique à partir des années 1960, période durant laquelle on a vu apparaître une lutte acharnée entre les chaînes de radio et de télévision pour les droits de diffusion, et entre les multinationales se disputant le statut de partenaire commercial des Jeux (Horne et Manzenreiter 2006). Ensuite, l'apparition de la télévision par satellite dans les années 1970 et 1980 a rendu les JO accessibles à un nombre sans précédent de spectateurs à travers le monde (Roberts 2004 : 114; Horne 2007 : 83).

Aujourd'hui, on assiste à une globalisation des Jeux et à une organisation toujours plus onéreuse, en lien avec la commercialisation et l'expansion de nouveaux moyens d'information de masse, en particulier numériques. Ces technologies se sont largement imposées, pour la première fois, aux JO de Pékin en 2008 (Chappelet et KüblerMabbott 2008 : 78-80, Marshall et al. 2010), les Jeux devenant alors des événements médiatiques à grande échelle, et attirant un public composé non seulement de téléspectateurs, mais aussi d'utilisateurs d'Internet.

Pour certains chercheurs, le processus de «médiatisation » des JO, à l'issue duquel des compétitions purement sportives se transforment en spectacle médiatique mondial (McDaniel et Sullivan 1998 ; Rowe 2004), modifie en profondeur la nature-même de l'événement ${ }^{13}$.

Selon Axel Bruns (Bruns 2008), la production et la consommation de médias se sont radicalement transformées, dans la mesure où les utilisateurs ont désormais accès à de nombreuses alternatives aux usages traditionnels. Bruns propose

13 Pour Hutchins et Mikosza, ce processus implique « a broader ontological shift in terms of how the social world is perceived institutionally and individually » (Hutchins et Mikosza $2010: 283$ ). 
d'examiner ce modèle de consommation dans le cadre de la théorie du prod-usage qui suppose un refus des médias traditionnels et du rôle passif du spectateur, au profit des nouveaux médias et d'un rôle actif pour les utilisateurs dans la production et l'utilisation de l'information (Bruns 2008 : 16 ; Vann 2014 : 440-441). Dans ce cadre, la consommation de médias traditionnels est complétée par le recours aux nouveaux médias. En effet, avec le développement du Web 2.0, les nouveaux médias acquièrent des fonctions uniques en matière de couverture de l'information. Leur existence est purement numérique, sans aucun analogue papier ni autre support matériel. Leur caractère interactif permet également aux utilisateurs d'entrer en communication, de modifier toute information, de la compléter et de l'adapter à leur représentation du monde (Lossevskaja 2013). Outre ces caractéristiques, les nouveaux médias se distinguent par la fragmentation de leur auditoire. Contrairement aux téléspectateurs qui ne peuvent recevoir l'information qu'à travers un canal unique, les utilisateurs des nouveaux médias se répartissent en communautés regroupées autour de centres d'intérêt communs et peuvent accéder aux informations les plus variées. Un tel environnement médiatique étant considéré comme plus difficile à contrôler que des retransmissions télévisées, les nouveaux médias ont souvent été décrits comme un espace propice à la formation de discours médiatiques alternatifs ${ }^{14}$.

\subsection{Les J0 au prisme des nouveaux médias}

Si les droits de diffusion télévisuelle des Jeux Olympiques sont détenus par le Comité international olympique (CIO), des textes circulant sur Internet et n'ayant pas toujours d'auteur clairement identifié sont susceptibles d'échapper à tout contrôle et peuvent ainsi constituer un discours médiatique indépendant. Dès lors, pour tenter de pallier ce manque de contrôle, les organisateurs officiels utilisent activement les technologies numériques et tentent de centraliser les sources d'information alternatives isolées (McGillivray $2013: 5$ ).

Au cours de leur histoire, les Jeux Olympiques ont, comme évoqué précédemment, principalement été couverts par les médias traditionnels. Internet a d'abord été utilisé pour transmettre l'information plus rapidement que via la presse, la radio ou la télévision. $\mathrm{Si}$, à la fin du $\mathrm{XX}^{\mathrm{e}}$ siècle, la sphère du web pouvait offrir un point de vue alternatif sur les JO, elle ne représentait qu'une source très marginale, dans un paysage médiatique dominé par la télévision. Ainsi, au début des années 2000, les principaux usages d'Internet se résumaient à la publication de résultats, de comptes

14 De nombreuses fonctions d'émancipation ont ainsi été prêtées aux nouveaux médias, de la promotion de la diversité culturelle (Papa 2012 : 2219-2220) à l'amélioration des conditions sociales et démocratiques (Sandvoss 2004 : 40). Les nouveaux médias ont également été présentés comme un facteur possible de libéralisation du régime en Russie (Nocetti 2013). 
rendus des compétitions, de photos, d'enregistrements audio et vidéo, consultés par un très faible pourcentage de téléspectateurs (Sandvoss $2004: 42$ ). Les organisateurs des JO ont commencé à se tourner vers Internet au début du XXI siècle, ébranlant l'hégémonie de la télévision. Les Jeux de Pékin en 2008 sont les premiers à avoir été entièrement diffusés en ligne, en plus de la couverture télévisuelle traditionnelle (Latham 2012), suivis par les Jeux de Vancouver en 2010, et de Londres en 2012. Le Comité d'organisation de Sotchi 2014 a aussi choisi d'assurer la retransmission en ligne des compétitions, comptant sur une fréquentation de plus de 100 millions de spectateurs sur le site officiel des Jeux (Olimpijskie igry Soči 2014).

L'apparition des médias sociaux a entraîné une restructuration du secteur économique des médias sportifs, en remettant par exemple en cause les principes de retransmission centralisée des méga-événements. Comme évoqué précédemment, l'interactivité est la caractéristique essentielle de ces médias qui, en offrant aux utilisateurs la possibilité de modifier et commenter le contenu, en font les destinataires et les producteurs d'information dans une évolution constante. Le système d'information sur les JO cesse ainsi d'être « fermé » (Hutchins et Mikosza $2010: 281$ ).

Avec l'évolution des nouveaux médias, la distinction entre discours médiatique officiel et discours non officiel est moins liée au contexte de production. En règle générale, les canaux d'information officiels privilégient la télévision parmi les médias traditionnels et les sites d'information parmi les nouveaux médias. Par exemple, le Comité d'organisation de Sotchi 2014 dispose d'un site internet officiel, <www. sochi2014.com>. Toutefois, pendant la préparation des Jeux, des sites non officiels dédiés aux problèmes liés à l'organisation et au déroulement des $\mathrm{JO}^{15}$ étaient également accessibles en Russie, l'identité des auteurs et des propriétaires de ces sites étant difficile à déterminer. En même temps, la popularité accrue des médias sociaux, initialement utilisés dans le cadre de communications informelles et de débats officieux, a commencé à attirer l'attention des organisateurs des JO qui s'en sont emparés, comme en témoigne l'existence sur Facebook de groupes officiels des JO de Londres et de Sotchi. De la même façon, en plus des communautés informelles, le réseau social Vkontakte dispose d'un groupe officiel sur les Jeux de Sotchi, accessible via un lien qui figure sur le site officiel des Jeux (Igry Soči 2014). Ainsi, le discours médiatique officiel se construit autour du site principal du Comité d'organisation et est relayé par les médias sociaux. 


\section{2. "Hot.Cool.Yours. " : Internet comme support du discours officiel sur les JO de Sotchi}

\subsection{Le site officiel, vitrine des JO}

La campagne officielle d'information consacrée aux Jeux de Sotchi a été lancée dans les médias sociaux en 2005, avant même que le CIO ait décidé de la ville qui accueillerait l'événement. Le site internet www.sochi2014.ru de la campagne officielle fut présenté par le Comité d'organisation des Jeux de Sotchi comme le rendez-vous médiatique le plus important. Pendant la campagne qui a duré de 2005 à 2014, le site a constamment subi des modifications, aussi bien techniques qu'en termes de contenu (Lossevskaja 2013). " Hot.Cool.Yours. », dont les points utilisés après chaque mot font explicitement référence à l'univers de la toile, se présente comme le slogan central, utilisé aussi bien par les médias traditionnels que par les nouveaux médias.

La version actuelle du site officiel puise dans le registre du Web 2.0, offrant des liens vers les groupes officiels dans les médias sociaux (Vkontakte, Facebook), ou vers d'autres outils ou plateformes d'information comme Twitter ou Google+. Toutefois, le site est constitué en majorité d'une offre d'informations stable, les possibilités en matière d'interaction étant assez limitées. Par exemple, il est totalement impossible de commenter directement les contenus et aucun lien vers des blogs n'est proposé. L'un des rares éléments interactifs est la rubrique « sondages ».

Sur le site officiel, les composantes sémantiques principales du discours sur les JO sont contenues dans les liens qui associent les Jeux à la culture, le « développement durable » et la notion d' « héritage ». L'expression « Olympiade culturelle » suppose en effet un lien entre les JO et le développement culturel et renvoie aux événements organisés pour montrer la diversité culturelle de la Russie (Olimpijskie igry Soči 2014). La notion de « développement durable», reprise par le comité d'organisation, intègre des éléments aussi disparates qu'un « mode de vie sain », «l'harmonie avec la nature », « un monde sans frontières », « la prospérité économique », « les technologies de pointe », « la culture et les valeurs nationales ». L'argumentation qui accompagne chacun de ces éléments de discours vise à démontrer les bénéfices économiques et sociaux liés au choix de Sotchi comme ville hôte des Jeux (Olimpijskie igry Soči 2014). Enfin, l'idée d'héritage est liée aux « changements positifs » que l'organisation des JO est censée apporter « en profondeur dans les domaines social, économique et écologique ». Parmi ces changements, on peut mentionner la construction des sites olympiques, la mise en œuvre d'un nouveau système de transports, ou encore la réintroduction de la panthère de Perse dans le Caucase, trois aspects particulièrement mis en avant. L'héritage immatériel, quant à lui, regroupe : 
l'accumulation des savoirs, des acquis et des expériences ; le développement du patriotisme et de la fierté nationale ; l'amélioration de l'image du pays et de la ville hôtes ; l'amélioration des relations sur la scène internationale ; davantage de tolérance et d'inclusion (entre autres pour les personnes souffrant d'un handicap physique), ainsi que la responsabilité sociale et économique (Olimpijskie igry Soči 2014).

L'accent est mis particulièrement sur le fait que, grâce à Sotchi, en plus de la dimension sportive, le rapport aux personnes handicapées est en train de changer dans l'ensemble du pays (Olimpijskie igry Soči 2014).

Tout cela contribue à la construction d'un discours de marque des JO de Sotchi. Les organisateurs mettent en avant le caractère innovant de ces Jeux à travers neuf termes visant à symboliser l'événement : perfection, efficacité, intégration, hospitalité, unité, originalité, respect, héritage et harmonie. Les Jeux de Sotchi ont ainsi vocation à devenir le lieu d'un dialogue des cultures substantiel et pacifique. L'événement lui-même, désigné comme " les Jeux de l'innovation » est présenté comme « une fête riche en opportunités, une source de croissance, de transformation et d'accomplissement» (Olimpijskie igry Soči 2014). Dans cette optique, le discours officiel, tel qu'il se donne à voir sur le site du comité d'organisation de Sotchi 2014, se construit principalement autour de l'idée de diversité culturelle et de la mise en avant des effets foncièrement positifs des Jeux. Le contrôle exercé sur l'accès au site et sur son contenu empêche toute sémantique négative.

\subsection{Le groupe officiel sur Vkontakte, prolongement du discours officiel}

Les médias sociaux, et notamment les communautés officielles créées autour des JO sur Vkontakte, théoriquement ouverts et non soumis aux contrôles, présentent en réalité un discours similaire au site d'information officiel.

Le groupe officiel du Comité d'organisation de Sotchi 2014 sur Vkontakte, auquel renvoie le site officiel, rassemble plus de 68 millions d'adhérents ${ }^{16}$. Il propose 26 thèmes de discussion, compte plus de 1800 posts sur son mur ouvert aux commentaires, ainsi que des matériaux visuels - albums photos, vidéos -, pouvant également tous être commentés. Pour les organisateurs, le but du groupe est « l'échange sur tout thème lié d'une façon ou d'une autre aux JO, au sport ou au statut de bénévole pendant les jeux » (Igry Soči 2014). Le ton général des conversations peut être qualifié de neutre et positif. L'intérêt porte surtout sur des questions comme : comment peuton participer aux Jeux en tant que volontaire ? Où en est l'itinéraire de la flamme olympique et quelles localités traverse-t-elle ? Comment obtenir des billets pour les différentes compétitions ? Les seuls commentaires négatifs concernent des aspects purement pratiques : «Pas évident de trouver des billets. C'est ma troisième nuit 
blanche d'affilée pour en obtenir deux ». Une place particulière est accordée à la rhétorique patriotique, présente notamment sous forme de petits poèmes tels que :

Les cieux se parent de rouge, de blanc, de bleu,

La Russie est avec nous - nous votons oui ! Et chacun peut le dire, à la terre entière : ces Jeux, nous les avons mérités toi et moi.

Beauté de l'Olympique Sotchi : neiges bleutées, huttes, nuits étoilées en mer Noire, montagnes, soleil, tout est pour toi! ${ }^{17}$ (Igry Soči 2014).

Dans son ensemble, le discours de la communauté olympique sur Vkontakte relaie les principales idées du site officiel de Sotchi 2014 au travers d'images romantiques d'un public gagné à la cause patriotique : malgré son caractère théoriquement ouvert, le groupe officiel sur Vkontakte fait l'objet de mécanismes de contrôle quant au contenu de ce qui s'y écrit.

\subsection{Le site officiel des fans : entre éloges et " critique constructive "}

Les fans des JO disposent également de leur site officiel qui propose, outre l'emblème et l'hymne des Jeux, l'accès à des résumés de l'actualité et des fiches sur les installations et les athlètes (Naša Olimpiada 2014). Le site n'a pas d'auteur désigné, mais une analyse de discours laisse supposer qu'il sert de complément au site officiel des Jeux, textes et visuels attestant du bon déroulement des préparatifs. Néanmoins, au sein d'un discours globalement positif, on retrouve quelques critiques qui concernent notamment les questions environnementales, les conditions des travailleurs immigrés, et les cas de violation des droits des habitants : " Vous voyez, tout n'est pas aussi rose qu'à la télévision » (Naša Olimpiada 2014). Si les critiques visent des problèmes inhérents à la préparation d'un événement de cette ampleur, elles ne s'attachent pas au contexte politique et social des JO de Sotchi. Ainsi ce site, non interactif et contrôlé, soutient-il globalement le discours officiel sur les JO, malgré une rhétorique qui propose parfois « une critique constructive ». On trouve une critique plus acerbe sur les sites d'information alternatifs.

« Красным, белым и синим светят небеса, С нами вместе Россия - голосуем за ! И всей планете сказать об этом может с нами любой - Игры, которые мы заслужили вместе с тобой ». « Красота Олимпийского Сочи : Голубые снега, терема, Черноморские звёздные ночи, Горы, солнце, всё-всё для тебя ! » 


\section{3. «Le festin au temps de la peste » : le discours non officiel en ligne}

\subsection{Des sites internet supports d'une critique virulente}

Sites internet indépendants, $\operatorname{blog} s^{18}:$ plusieurs supports digitaux relaient, quelques années avant les Jeux ${ }^{19}$, un discours alternatif abordant différentes questions dont la sécurité, les violations des droits des Tcherkesses dans la région, la corruption et les abus de pouvoir commis par les fonctionnaires russes. Par exemple, sur un des sites qui qualifie la région de Sotchi d' " Irak russe », on peut lire que cet événement aurait dû se dérouler « dans un pays sûr, n'étant pas en guerre et n'exterminant pas sa propre population » (Anti Soči 2014). L'idée même d'une Russie pays hôte des Jeux suscite des critiques, comme le montre le post suivant :

Il est absolument immoral d'autoriser l'organisation de Jeux Olympiques, dont la vocation est de promouvoir la paix et la coopération internationale à travers le sport, dans un pays aussi hypocrite que la Russie, qui a reconnu des génocides commis ailleurs tandis qu'elle nie toujours le génocide qu'elle a perpétré elle-même contre les Tcherkesses (No Soči 2014).

Enfin, ils attirent l'attention sur le niveau anormalement élevé des dépenses ainsi que sur les problèmes d'environnement et de corruption :

Les JO de Sotchi, c'est le détournement des fleuves ${ }^{20}$ d'aujourd'hui, aussi absurde, nocif, coûteux que le fut ce grandiloquent projet soviétique (STOP Olimpiade - 2014 v Soči).

Parmi les sites non thématiques diffusant un discours non officiel, on retrouve celui de l'opposant politique Boris Nemtsov ${ }^{21}$. En mai 2013, Boris Nemtsov et Leonid Martyniouk publient un rapport d'expertise intitulé Des Jeux d'hiver sous les

18 Voir par exemple <http://antisochi2014.wordpress.com>, <http://www.nosochi2014. com $>$ et $<$ http://nolympicgames.livejournal.com $>$.

19 Notons que la majorité des ressources en question n'a pas été mise à jour depuis 2010 ou 2011.

20 Cette évocation fait référence au grand projet soviétique de détournement des fleuves sibériens vers l'Asie centrale. Ce projet faisait partie du Programme d'amélioration de l'agriculture, adopté par le présidium du Parti communiste d'Union soviétique en 1965. Activement débattu dans les années 1970-80, il sera finalement abandonné. Voir Jérémy Allouche, "Continuité et discontinuité dans la politique de l'eau en Asie centrale », Cahiers d'Asie centrale, 2004, n 13/14, pp. 285-300.

$21<$ http://www.nemtsov.ru>. Boris Nemtsov a été assassiné à Moscou alors qu'il rentrait chez lui le 27 février 2015. 
Tropiques $^{22}$. Dans ce rapport, ils attribuent clairement le choix de Sotchi au président Vladimir Poutine, venu en personne au Guatemala où eut lieu le vote du CIO :

Les Jeux Olympiques d'hiver de Sotchi sont le projet personnel de Poutine. Ce dernier a conçu les Jeux comme son triomphe personnel, comptant sur le fait que la venue d'athlètes du monde entier entérinerait son leadership aussi bien en Russie que dans le monde entier (Nemcov et Martynjuk 2013).

Selon les auteurs, les principaux problèmes de ces Jeux sont les dépenses démesurées, la corruption, le flou du budget et les dégâts environnementaux :

« Un record est déjà établi pour ces Jeux : celui des pots-de-vin et du détournement des fonds publics » (Nemcov et Martynjuk 2013).

Dans son ensemble, ce discours repose sur le lien entre les Jeux de Sotchi et une augmentation des risques, qu'ils soient technologiques, écologiques ou terroristes.

On retrouve également un discours critique sur le site Ènciklopedija trat («Encyclopédie des dépenses »), créé par le Fonds de lutte contre la corruption d'Alekseï Navalny ${ }^{23}$. Son but est de présenter « une analyse détaillée des dépenses liées aux Jeux » (Ènciklopedija trat 2014). Le site propose de courtes fiches d'information et d'évaluation concernant les infrastructures et les montants des dépenses présumées. Ainsi l'aéroport international de Sotchi fait-il l'objet d'une bonne appréciation pour son fonctionnement, mais est très mal noté du point de vue de sa construction et de son inauguration. De nombreux exemples mettent en lumière la corruption des fonctionnaires, l'incompétence ou la négligence des responsables (Ènciklopedija trat 2014).

Ainsi les discours présents sur ces sites, particulièrement virulents, nous permettent de les classer dans la catégorie des discours non officiels, sur des sites non contrôlés et non interactifs.

\subsection{Les discours non officiels sur Vkontakte : des avis contrastés}

Enfin, comparons le discours internet officiel avec le discours non officiel présent sur le réseau social Vkontakte. Ce dernier se présente en effet comme un terrain privilégié pour les discussions non officielles sur les Jeux, tandis que l'activité des blogs et sites thématiques dédiés à la critique de l'événement est presqu'au point mort. Le plus grand groupe sur Vkontakte, « Olimpiada Soči 2014. The Informal Group »²,

22 Zimnjaja Olimpiada $v$ subtropikah, disponible sur $<$ http://nemtsov.ru/2013/05/ zimnyaya-olimpiada-v-subtropikax/>.

$<$ http://sochi.fbk.info/ru/>. 
accueille des discussions notamment sur la répartition des moyens financiers et sur l'implication personnelle de Vladimir Poutine dans la mise en place des Jeux. Ainsi, dans les commentaires d'une interview donnée par un membre du CIO sur la corruption liée aux Jeux et sur les « relations publiques » de Vladimir Poutine, on retrouve la remarque suivante :

L'important, c'est que nous remportions des médailles à Sotchi. Ce qui a été volé importe peu!

Des commentaires montrent également les protestations exprimées sur des pancartes lors du passage de la flamme olympique dans certaines villes russes comme :

Le Pillathlon ${ }^{25}$, le sport le plus répandu aux Jeux de Sotchi !

Détournement par équipe et course de corruption, voilà les sports les plus populaires des JO!

Les informations concernant ces protestations sont accompagnées de commentaires tantôt critiques, tantôt en faveur des JO :

Les Jeux Olympiques volent l'argent des enfants handicapés, des femmes enceintes, des pensionnés...

Ils ont toujours volé et continueront à voler. Au moins, les Jeux auront permis de remettre de l'ordre dans ce poulailler qu'on appelle Sotchi.

Personne n'a rien volé à personne. Toutes les infrastructures sportives appartiennent à des entreprises [...] et ces entreprises, bien qu'elles soient officiellement biens d'État, ont des actionnaires qui se partagent les bénéfices.

Les autorités ne sont pas aussi propres sur elles qu'elles le voudraient. Mais elles sont quand même sur la bonne voie. Nos enfants seront fiers de nous (Olimpiada Soči 2014).

On constate le même rapport ambigu aux JO au travers de commentaires comme :

Les Jeux Olympiques sont un grandiose divertissement qui a englouti d'énormes sommes d'argent qui auraient dû financer des besoins autrement plus criants du pays. On aurait pu organiser des JO quelques années plus tard, sans dilapider ces sommes folles en détournements de fonds et dessous-de-table. Et à un autre endroit, loin des agglomérations, pour que la population n'en pâtisse pas (Olimpiada Soči 2014).

Le relais de la flamme olympique, qui a fait le tour de la Russie au cours des mois précédant l'ouverture des Jeux, a également fait couler beaucoup d'encre sur

25 Le terme russe initialement utilisé est « Spërling » (Спёрлинг), jeu de mots entre la discipline olympique du Curling et le verbe argotique «speret' (cnepemb) qu'on peut traduire par « chourer » ou « piquer». 
Vkontakte. Couvert par tous les médias de masse, ce relais a suscité des commentaires de toutes sortes, le plus souvent critiques :

On n'avait encore jamais vu pareille lubie nulle part. Ils la baladent comme un idiot baladerait sa balalaïka.

Combien d'argent a-t-il fallu dépenser pour trimballer cette satanée flamme aux quatre coins du pays ? C'est un scandale ! Cet argent aurait pu servir à sauver des vies, à payer des opérations chirurgicales et des traitements médicaux !

Les élus du peuple auraient mieux fait de s'occuper des gens, au lieu de vouloir en faire toujours plus! (Olimpiada Soči 2014).

Dans l'ensemble, les émotions exprimées par les membres de la communauté en question sont contrastées :

Je suis fier de pouvoir emmener mon fils aux Jeux Olympiques. Dans les années 80, c'est quelque chose que mes parents ne pouvaient pas se permettre.

Aux JO de Sotchi, ce qui me dérange le plus, ce sont ces insultes flagrantes au bon sens; l'insolence éhontée de ce qui se passe, et la mise en scène grossière d'une allégresse nationale.

Une mise en scène. J'aime la Russie et les gens qui y vivent. Et je suis triste pour nous tous. Ces Jeux Olympiques, c'est vous et moi, les contribuables, qui allons les payer!

Peu importe que les JO soient un moment festif dans le monde entier; aujourd'hui, en Russie, c'est un festin en temps de peste, et ceux qui paient ce festin n'ont pas de place à table (Olimpiada Soči 2014).

Tout comme dans l'ensemble du discours non officiel, les JO sont présentés sur Vkontakte comme un facteur d'aggravation des problèmes socio-économiques et politiques existant en Russie. En même temps, on observe un consensus au sein de l'ensemble des médias sociaux, officiels ou non, sur l'apport positif des JO comme facteurs de consolidation de la nation russe et promoteurs du patriotisme.

\section{Les JO vus par les Russes : des perceptions mitigées}

Pour comparer les discours internet sur les Jeux et les représentations des Jeux au sein de la population russe, nous nous référons aux résultats de sondages conduits en janvier-février 2014, avant le début des Jeux.

Les résultats d'un sondage du Centre russe d'étude de l'opinion publique (VTSIOM), publiés le 5 février 2014, nous éclairent sur les attitudes des citoyens russes vis-à-vis de Sotchi. Le supporter russe « type » y est décrit comme un homme jeune, vivant à Moscou ou Saint-Pétersbourg ; pour les citoyens, les Jeux sont 
essentiellement perçus comme une opportunité de voir des compétitions sportives : deux semaines avant l'ouverture des Jeux, $73 \%$ des citoyens russes comptaient bien les suivre ; $51 \%$ des citoyens russes comptaient bien sur la victoire de l'équipe nationale en nombre de médailles par pays. Le centre Iouri Levada a, quant à lui, mené une enquête plus détaillée entre le 24 et le 27 janvier 2014, incluant des questions sur la dimension financière des Jeux et proposant plusieurs réponses par question. Ce sondage révèle que $38 \%$ de la population considéraient que les Jeux étaient « un moyen facile de détourner de grosses sommes du budget de l'État», alors que $23 \%$ faisaient remarquer que les Jeux étaient « un événement important et prestigieux pour le pays, renforçant l'unité nationale » (Centre Levada 2014b).

Notons que ni le VTSIOM, ni le Centre Levada n'ont tenu compte de la répartition potentielle du public entre téléspectateurs et internautes, ce qui pourrait influer sur les différences de perception. Par ailleurs, si le sondage du Centre Levada révèle les principaux problèmes soulevés par la population, il recourt à des questions fermées qui ne permettent pas de refléter la diversité des opinions du public russe.

Le projet « Sotchi-2014, projet national $»^{26}$, a quant à lui privilégié la méthode des questions ouvertes. L'enquête s'est déroulée du 15 janvier au 6 février 2014 et 582 personnes y ont répondu dans sept agglomérations russes (dont 308 femmes et 274 hommes, âgés de plus de 18 ans). Les questions suivantes ont été posées : " Quel est votre principale source d'information sur les JO ? », « Quels mots associez-vous aux Jeux Olympiques de Sotchi ? » et «Que signifient pour vous les Jeux Olympiques de Sotchi ?»

Plus de $40 \%$ des personnes interrogées (245 personnes) ont désigné Internet comme leur principale source d'information. Il faut toutefois tenir compte du fait que 259 personnes ont été interrogées via Internet (via leur messagerie électronique, le réseau social Vkontakte, ou des applications de Google), ce qui constitue un filtre dans la sélection des répondants. 323 autres personnes ont répondu à un enquêteur. Parmi elles, 92 ont indiqué utiliser Internet pour s'informer sur les Jeux. Selon les résultats préliminaires, les utilisateurs d'Internet s'intéressant aux Jeux en janvier-février 2014 (avant l'ouverture de ces derniers, donc) représentaient environ $30 \%$ du public, tous médias confondus.

26 L'étude s'est déroulée dans les villes de Saint-Pétersbourg, Samara, Petrozavodsk, Velikie Louki (oblast de Pskov), Solikamsk (kraï de Perm), les villages de Lovozero (oblast de Mourmansk) et Gostilitsy (oblast de Leningrad) dans le cadre du « projet national Sotchi 2014 » du centre de recherche «Politique, société, sport : analyse comparative ", du Département d'études politiques comparatives de l'Institut NordOuest de gestion de l'Académie Présidentielle d'économie et d'administration publique, 15 janvier - 6 février 2014. 
Plus de la moitié des personnes interrogées (357) ont répondu aux questions sur ce que leur inspiraient les JO. 45 ont répondu que les Jeux ne représentaient rien pour eux, tandis qu'un grand nombre de réponses (312) se sont distribuées entre les catégories « Positif» $(49 \%)$, « Neutre » $(17 \%)$ ou « Négatif» $(34 \%)$ en fonction de leur connotation sémantique.

Parmi les connotations " neutres », on retrouve ce qui a trait aux différents sports d'hiver (« hockey », « biathlon »), aux symboles du mouvement olympique et des JO de 1980 et 2014 (« flamme olympique », « anneaux », « ours », « lapin », « léopard»), le slogan des Jeux (« hot, cool, yours »), des mots génériques renvoyant à la compétition («jeux », « événement sportif», « sportifs ») et des termes renvoyant à l'environnement naturel des Jeux (« hiver », « neige », « montagnes »).

L'analyse des réponses fournies (positives et négatives) permet d'isoler les composantes sémantiques centrales des perceptions russes sur les Jeux. Les personnes interrogées considèrent tout d'abord ces derniers comme « un événement important », « une grande fête sportive ». Le terme « fête » est celui qui revient le plus souvent (33 fois, soit plus de $10 \%$ des réponses détaillées). La notion de fierté est également centrale, donnant lieu à différentes associations : «fierté pour la Russie », " fierté pour les sportifs », " fierté pour la nation russe », « je suis très fier de la Russie ». Le terme « victoire » est le troisième à apparaître le plus souvent (6\%). Les connotations émotionnelles ( «joie », « émotion») ressortent aussi particulièrement, tout comme les avis qui associent l'événement à un facteur d'unification (« rassemblement », « union des peuples du monde entier »). Certaines personnes évoquent une influence positive des Jeux sur l'économie et le développement du sport en Russie, ainsi que sur le statut international du pays (« croissance de l'économie », « développement du sport dans le pays », « prestige », « ascension au niveau international »).

$\mathrm{Au}$ sein des réponses à connotation négative, les composantes sémantiques principales du discours renvoient à l'argent (mentionné plus de 50 fois, soit dans $15 \%$ des réponses détaillées) : " argent jeté par les fenêtres », " argent dilapidé », « dépenses inutiles ». Cette catégorie est particulièrement présente dans l'évaluation de la préparation des Jeux, associée aux expressions « blanchiment d'argent », " vol du budget », « corruption », « détournement de fric », « dilapidation du budget », « appropriation des deniers publics » ou « manne pour les voleurs $»^{27}$. Certaines personnes interrogées ont qualifié les Jeux de « mise en scène », de « fanfaronnade » et de « festin au temps de la peste ». Des réponses isolées évoquent les conséquences négatives des Jeux pour l'économie et la population russes (" problèmes environnementaux », « crise financière », " une fois de plus, on a dépouillé le

27 En russe, dans la version originale : « отмывание денег », « воровство бюджета », «коррупция », «распил бабла », « транжирство бюджета », « казнокрадство », « кормушка для воров ». 
peuple »), et donnent une opinion générale négative de l'événement («l'illusion d'un prestige de la Russie au niveau international », « une honte »).

L'analyse des réponses au sondage permet d'isoler les composantes sémantiques reprises dans le tableau 2. Ces résultats nous indiquent que les perceptions du public russe intègrent à la fois des éléments du discours médiatique officiel et non officiel. En effet, le discours olympique officiel se retrouve au sein des perceptions de l'opinion publique, qui voit les JO avant tout comme une fête, un facteur d'unité nationale et une source de fierté pour le pays et ses habitants qui «vont montrer au monde une nouvelle Russie forte et sportive ».

En ce qui concerne le discours médiatique non officiel, rappelons que dans son segment non interactif (constitué par les sites internet d'opposition), les JO sont présentés de façon négative, alors que dans le segment interactif (les communautés non officielles des réseaux sociaux), l'opinion est plus mitigée. D'un côté, les Jeux sont critiqués notamment pour les aspects négatifs liés à leur organisation, surtout au niveau financier, mais de l'autre, ces Jeux sont présentés comme une opportunité unique en termes de partage d'expérience et de symbole. On retrouve ces éléments du discours médiatique non officiel également dans la façon dont se construisent les représentations au sein de l'opinion publique russe, tiraillée entre une critique des dérives financières et la reconnaissance de l'importance symbolique des Jeux.

Tableau 1 :

Composantes sémantiques des perceptions par l'opinion publique russe des JO

\begin{tabular}{|c|c|c|c|}
\hline \multicolumn{2}{|c|}{ Les JO comme événement } & \multicolumn{2}{|c|}{$\begin{array}{c}\text { Les JO comme affirmation de l'identité } \\
\text { nationale }\end{array}$} \\
\hline+ & - & + & - \\
\hline $\begin{array}{c}\text { divertissement } \\
\text { spectacle } \\
\text { développement du sport } \\
\text { développement } \\
\text { économique } \\
\text { innovations }\end{array}$ & $\begin{array}{c}\text { dépenses d'argent } \\
\text { détournement } \\
\text { corruption } \\
\text { vol }\end{array}$ & $\begin{array}{c}\text { fierté nationale } \\
\text { fête } \\
\text { rassemblement } \\
\text { esprit patriotique } \\
\text { crise }\end{array}$ & $\begin{array}{c}\text { hostin en temps de peste } \\
\text { poudre aux yeux } \\
\text { mise en scène } \\
\text { humiliation }\end{array}$ \\
\hline
\end{tabular}

\section{Conclusion}

La comparaison entre le discours internet sur les Jeux et les résultats de sondages révèle des parallèles sémantiques entre les deux types de discours. Les appréciations positives et négatives se construisent toutes les deux autour des mêmes thématiques : les JO en tant que grand événement sportif et en tant que vecteur de construction de l'identité nationale (Gorokhov, 2013). Les divisions de l'opinion publique se 
révèlent donc profondes autant sur le thème des $\mathrm{JO}$ que sur des problèmes cruciaux du développement de la Russie en général, ces derniers étant principalement abordés via les segments interactifs des médias sociaux.

L'analyse du discours sur les JO dans les ressources internet russes montre que de façon générale, les nouveaux médias se divisent en groupes interdépendants en fonction du discours médiatique qu'ils construisent. Comme le montre le graphique 2, dans le système néo-autoritaire russe, on observe une convergence, repérée plus haut, entre médias traditionnels et nouveaux médias. Cette convergence est due à la fonction de télévision assumée de facto par certains sites internet. En particulier, le site officiel des JO fait fonction de « deuxième écran » de télévision (Hutchins et Rowe 2012), sans aucune interactivité (carré 1). Servant de transition vers l'interactivité pour les utilisateurs tout en restant dans le cadre d'un discours officiel positif, les nouveaux médias acquièrent les fonctions des médias traditionnels. Non interactifs, ils n'offrent pas aux internautes de possibilité de participation, et sont en outre contrôlés (carré 1). Les réseaux sociaux participent également à la mise en circulation d'un discours positif sur les JO qui se construit dans les segments interactifs et contrôlés des médias sociaux, telles les communautés officielles des Jeux sur Vkontakte (carré 3). Quant aux sites non officiels portant un jugement critique et permettant la formulation d'un discours médiatique non contrôlé, leur accès n'est pas interactif, ce que confirment les connotations exclusivement négatives sur les Jeux qui y sont publiées. Ces sites non officiels échappent certes au contrôle de leur contenu, mais n'offrent pas d'interactivité avec le public (carré 2). Ainsi, l'intersection entre discours officiel et non officiel, mis en lumière par les résultats des sondages, se réalise au sein des segments non contrôlés des réseaux sociaux à fonctionnement interactif - communautés non officielles sur les Jeux, dans Vkontakte.

L'influence réciproque que se livrent discours officiel et non officiel sur des sujets d'informations importants pour la société russe se donne donc à voir au sein des médias sociaux. Les restrictions d'accès aux médias sociaux et le contrôle de leur contenu passent par des processus de convergence - lorsque les médias sociaux acquièrent les fonctions des médias traditionnels, et de regroupement - lorsque se créent des groupes de médias inter-reliés, qu'ils soient interactifs ou non interactifs. 


\section{Graphique 2}

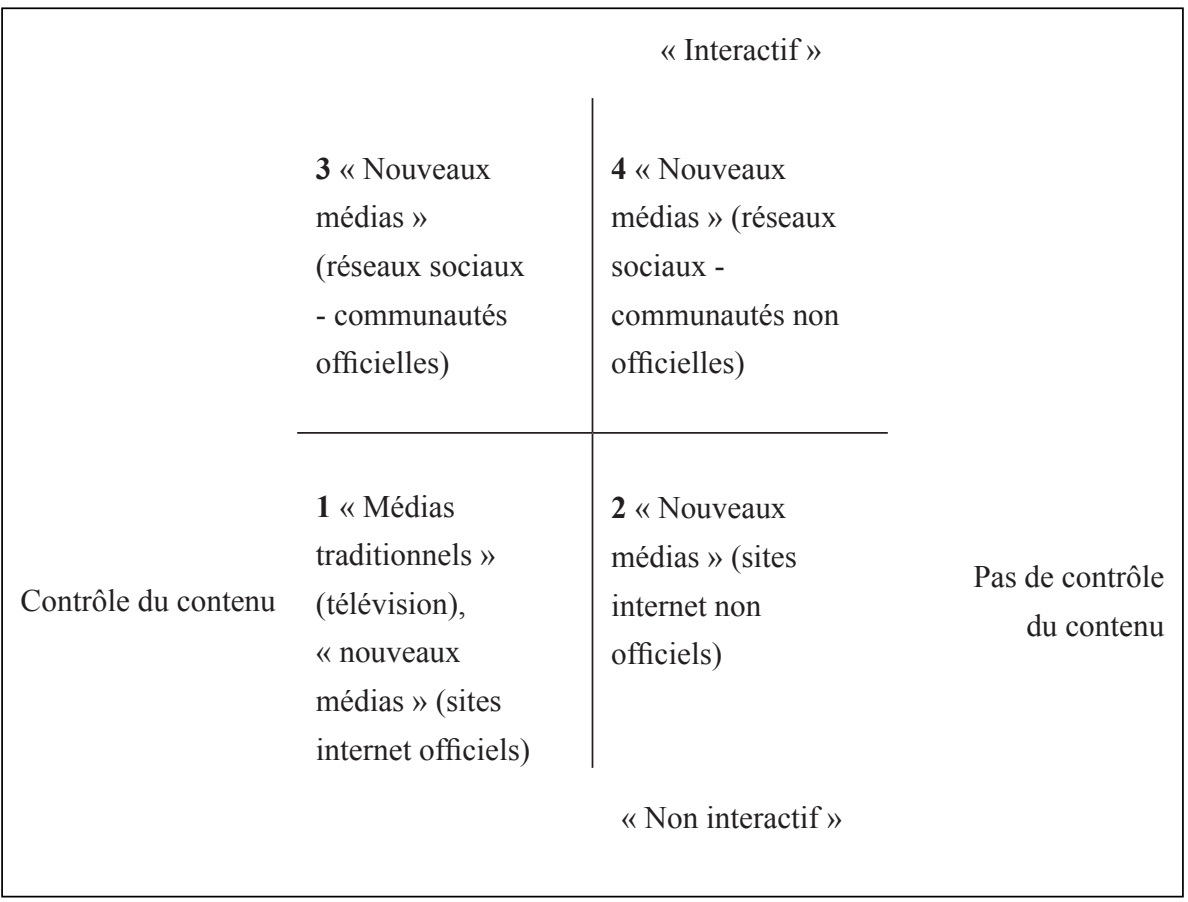

\section{Bibliographie}

Anti Soči 2014 : «Anti Soči 2014. Protiv grjaznoj politiki. Protiv Olimpiady v «russkom Irake» » (Anti-Sotchi 2014. Contre la sale politique. Contre les Jeux Olympiques dans l' «Irak russe ») document en ligne, consulté le 15.01.2014, <http://antisochi2014. wordpress.com/category/anti-sochi>.

Becker 2004 : Jonathan Becker, « Lessons from Russia : A Neo-Authoritarian Media System », European Journal of Communication, 2004, n 19, pp. 139-163.

Chappelet et Kuebler-Mabbott 2008 : Jean-Lou Chappelet et Brenda Kübler-Mabbott, The International Olympic Committee and the Olympic System, London, New York : Routledge, 2008.

Daucé 2014a : Françoise Daucé, " Les médias russes en ligne de mire », Huffingtonport.fr, 08.05.2015, document en ligne, consulté le 17.07.2015, <http:/www.huffingtonpost.fr/ francoise-dauce/propagande-medias-russie-ukraine_b_5285908.html $>$.

Daucé 2014b : Françoise Daucé, « Le journalisme en ligne en Russie : les jeux ordinaires du contrôle politique », Les Études du CERI, 2014, n² 203, avril 2014.

Ènciklopedija trat 2014 : Ènciklopedija trat 2014. Soči-2014: Analitičeskij otčet (L'Encyclopédie des dépenses 2014. Sotchi 2014. Compte-rendu analytique), Fond bor'by s korrupciej (Fond de la lutte anti-corruption), document en ligne, consulté le 10.11.2014, <http:// sochi.fbk.info/ru/>.

Findling et Pelle 2004 : John E. Findling et Kimberley D. Pelle, Encyclopedia of the Modern Olympic Movement Westport, CT, London : Greenwood Press, 2004. 
FOM 2014 : « Internet v Rossii : dinamika proniknovenija. Vesna 2014 » (« La diffusion d'internet en Russie (Fondation pour l'analyse de l'opinion publique), printemps 2014 »), Fond obščestvennogo mnenija, document en ligne, consulté le 10.11.2014, $<$ http://runet.fom.ru/Proniknovenie-interneta/11567>.

Gorohov 2013 : Vladimir A. Gorohov, « Nacional'nye identičnosti v global'noj sportivnoj kul'ture : Vyzovy Soči-2014 dlja olimpijskoj komandy Rossii » (Les identités nationales dans la culture sportive mondiale : l'appel de Sochi 2014 pour l'équipe olympique de Russie), Žurnal sociologii i social'noj antropologii, 2013, vol. XVI, n 5, Tematičeskij nomer « Sportivnye mega-sobytija v politike, obšestve i kul'ture » (numéro thématique «Les méga-événements sportifs : aspects politiques, sociaux, culturels), pp. 71-86.

Horne 2007 : John Horne, « The Four "Knowns" of Sports Mega-Events », Leisure Studies, 2007, vol 26, $\mathrm{n}^{\circ} 1$, pp. 81-96.

Horne et Manzenreiter 2006 : John Horne et Wolfram Manzenreiter (eds.), Sport Mega-Events : Social Scientific Analyses of a Global Phenomenon, Malden, MA, Oxford : Blackwell, 2006.

Hutchins et Mikosza 2010 : Brett Hutchins et Janine Mikosza, «The Web 2.0 Olympics. Athlete Blogging, Social Networking and Policy Contradictions at the 2008 Beijing Games », Convergence: The International Journal of Research into New Media Technologies, 2010, vol. 16, n 3, pp. 279-297.

Hutchins et Rowe 2012 : Brett Hutchins et David Rowe, Sport Beyond Television. The Internet, Digital Media and the Rise of Networked Media Sport, New York, London, Routledge, 2012.

Igry Soči 2014 : «Igry Soči 2014. Oficial'noe soobščestvo Vkontakte » (Les Jeux de Sotchi 2014. Communauté officielle sur Vkontakte), document en ligne, consulté le 13.01.2014, $<\mathrm{http}: / / \mathrm{vk} . \mathrm{com} /$ sochi2014>.

Jennings 2012 : Will Jennings, Olympic Risks, Basingstoke and New York : Palgrave Macmillan, 2012.

Koltsova 2006 : Olessia Koltsova, News Media and Power in Russia, Londres : Routledge, 2006.

Oates 2007 : Sarah Oates, « The neo-Soviet model of the media », Europe-Asia Studies, 2007, vol. 59, n 8, pp. 1279-1297.

Latham 2012 : Kevin Latham, «Unimagined China: Media, Technologies and the Fragmentation of National Olympic Audiences », The International Journal of the History of Sport, 2012, vol. 29, n 16 , pp. 2311-2325.

Lee 2008 : Hongsub Lee, « Progress or Regress in Russian Mass Media ? », International Area Studies Review, 2008, vol. 11, n 2, pp. 231-243.

Levada-centr 2014a : «Internet ne smog zamenit' rossijanam TV v kačestve istočnika informacii, Levada-centr » (L'internet n'a pas réussi à remplacer la télévision comme source d'information pour les Russes), document en ligne, consulté le 10.11.2014, $<$ http://www.levada.ru/18-06-2014/internet-ne-smog-zamenit-rossiyanam-tv-vkachestve-istochnika-informatsii>.

Levada-centr 2014b : «Zimnie Olimpijskie igry v Soči : interes, nadeždy i ocenka, Levadacentr » (Les JO d'hiver à Sochi : intérêt, espoirs, et perception, Centre Levada) document en ligne, consulté le 11.11.2014, <http://www.levada.ru/05-02-2014/zimnieolimpiiskie-igry-v-sochi-interes-nadezhdy-i-otsenka>.

Losevskaja 2013 : Ekaterina N. Losevskaja, « Rol' 'novyh media' v informacionnoj kampanii 'Soči-2014' " (Le rôle des 'nouveaux médias' dans la campagne médiatique 'Sotchi 2014'), Žurnal sociologii i social'noj antropologii, vol. XVI, n ${ }^{\circ}$, Tematičeskij nomer 
« Sportivnye mega-sobytija v politike, obšestve i kul'ture » (numéro thématique « Les méga-événements sportifs : aspects politiques, sociaux, culturels »), pp. 203-220.

Marshall, Walker et Russo 2010 : David Marshall, Becky Walker et Nicolas Russo, « Mediating the Olympics », Convergence : The International Journal of Research into New Media Technologie, 2010, vol. 15, n 3, pp. 263-278.

McDaniel et Sullivan 1998 : Stephen McDaniel et Christopher Sullivan, « Extending the Sports Experience : Mediations in Cyberspace », in Wenner L. (ed.) Mediasport, London : Routledge, 1998, pp. 266-28.

McGillivray 2013 : David McGillivray, « Digital Cultures, Acceleration and Mega Sporting Event Narratives », Leisure Studies, document en ligne, consulté le 10.01.2014, <http:// dx.doi.org/10.1080.02614367.2013.841747>.

Na starte Olimpiady 2014 : « Na starte Olimpiady 2014 » (« Au début de l’Olympiade 2014 »), Vserossijskij centr obščestvennogo mnenija (VCIOM), document en ligne, consulté le 12.11.2014, <http://wciom.ru/index.php?id=459\&uid=114700>.

Naša Olimpiada 2014 : « Naša Olimpiada, Oficial'nyj sajt fanatov Olimpiady v Soči 2014. Olimpijskie igry v Soči - Žarkie. Zimnie. Tvoi ! » (Notre Olympiade, site officiel des fans de l'Olympiade de Sotchi 2014. Les JO à Sotchi - Hot. Cool. Yours !), document en ligne, consulté le 14.01.2014, < http://наша-олимпиада.pф>.

Nemcov et Martynjuk 2013 : Boris Nemcov et Leonid Martynjuk, Zimnjaja Olimpiada v subtropikah (Des Jeux d'hiver sous les Tropiques), document en ligne, consulté le 15.01.2014, <http://www.nemtsov.ru/?id=718789>.

No Soči 2014 : « No Sochi 2014. 14 pričin protivostojanija planam provedenija Olimpiady Soči -2014» (Non à Sotchi 2014 : 14 raisons de s'opposer à l'organisation des Jeux Olympiques à Sotchi -2014), document en ligne, consulté le 15.01.2014, <http:// www.nosochi2014.com/campaign/14-prichin-protivostoyaniya-planam-provedeniyaolimpiadi-sochi-2014.php>.

Nocetti 2013 : Julien Nocetti, "Internet, accélérateur des transformations de la Russie », Études, 2013, n 2, pp. 151-160.

Olimpiada Soči 2014 : « Olimpiada Soči 2014. Neformal'naja gruppa Vkontakte » (Les Jeux Olympiques de Sotchi 2014. Groupe informel sur Vkontakte), document en ligne, consulté le 15.01.2014, <http://vk.com/stranica_01>.

Olimpijskie igry Soči 2014 : «Olimpijskie igry Soči 2014. Oficial’nyj sajt » (Les Jeux Olympiques de Sotchi 2014. Site officiel), document en ligne, consulté le 13.01.2014, $<$ http://www.sochi2014.com>.

Papa 2012 : Françoise Papa, « Global Media Events : Communication Strategies, Social Network Patterns and Propaganda Models - A Complex and Challenging Reconciliation », The International Journal of the History of Sport, 2012, vol. 29, n 16, pp. 2218-2230.

Proulx et Latzko-Toth 2000 : Serge Proulx et Guillaume Latzko-Toth, « La virtualité comme catégorie pour penser le social : l'usage de la notion de communauté virtuelle » Sociologie et sociétés, 2000, vol. 32, n², pp. 99-122.

Proulx 2006 : Serge Proulx, « Communautés virtuelles : ce qui fait lien » in Proulx S., Poissant L. et Sénécal M., Communautés virtuelles : penser et agir en réseau, Québec : Presses de l’Université Laval, 2006, pp. 13-26.

Roberts 2004 : Ken Roberts, The Leisure Industries, Basingstoke and New York : Palgrave Macmillan, 2004.

Roche 2000 : Maurice Roche, Mega-Events and Modernity: Olympics and Expos in the Growth of Global Culture, London : Routledge, 2000. 
Rowe 2004 : David Rowe, Sport, Culture and the Media : The Unruly Trinity, Maidenhead : Open University Press, 2004.

Sandvoss 2004 : Cornel Sandvoss, « Technological Evolution or Revolution? Sport Online Live Internet Commentary as Postmodern Cultural Form », Convergence : The International Journal of Research into New Media Technologies, 2004, vol. 10, n 3, pp. 39-54.

STOP Olimpiade-2014 v Soči : « STOP Olimpiade-2014 v Soči » (Non aux Jeux Olympiques 2014 à Sotchi), document en ligne, consulté le 15.01.2014, <http://nolympicgames. livejournal.com>.

Vann 2014 : Portia Vann, « Changing the Game : The Role of Social Media in Overcoming Old Media's Attention Deficit Toward Women's Sport », Journal of Broadcasting and Electronic Media, 2014, vol. 58, n³, pp. 438-455.

VKontakte 2014 : «About VKontakte », VKontakte, document en ligne, consulté le 15.11.2014, $<$ http://vk.com/about>. 